\title{
A Miniature Instrument Tip Force Sensor for Robot/Human Cooperative Microsurgical Manipulation with Enhanced Force Feedback
}

Peter J. Berkelman, Louis L. Whitcomb, Russell H. Taylor, and Patrick Jensen

Engineering Research Center for Computer Integrated Surgical Systems and

Technology and Microsurgery Advanced Design Lab

Johns Hopkins University

Baltimore, MD 21218*

\begin{abstract}
This paper reports the development of a new miniature force sensor to measure forces at the tip of a microsurgical instrument in three dimensions with sub-millinewton resolution. This sensor will enable enhanced force feedback during surgical intervention in which a user directly manipulates surgical instruments cooperatively with a forcereflecting robot arm. This "steady-hand" scaled force interaction enables a surgeon to sense millinewton forces between the instrument and delicate body tissues during microsurgery which would otherwise be far below the threshold of human tactile sensing. The magnified force feedback can increase the dexterity of the surgeon and improve safety by preventing large damaging forces from being exerted by the instrument. The design and analysis of the new force sensor is presented with preliminary testing and force scaling control results.
\end{abstract}

Keywords: robotics and robotic manipulators, MEMS based medical devices, clinical human computer interfaces

\section{Introduction}

The limits of microsurgical procedures are presently defined by the visual acuity, dexterity, and tactile sensitivity of the surgeon. We have developed a compact robot assistant manipulator arm designed to enhance human tactile sensitivity and dexterity in microsurgical operations. Our goal is to enable new procedures to be performed and to improve measurable outcomes of microsurgery.

The robotic system is based on the "steady-hand" cooperative manipulation paradigm in which the surgeon and the robot both hold a microsurgical instrument and the robot end effector responds directly to comply to the sensed manipulation forces of the surgeon's hand [1, 2]. Direct hand-guided robot manipulation for surgery has been investigated by a number of authors $[3,4,5]$. Our

\footnotetext{
* We gratefully acknowledge the support of the National Science Foundation under grants IIS9801684 and EEC9731478.
} 


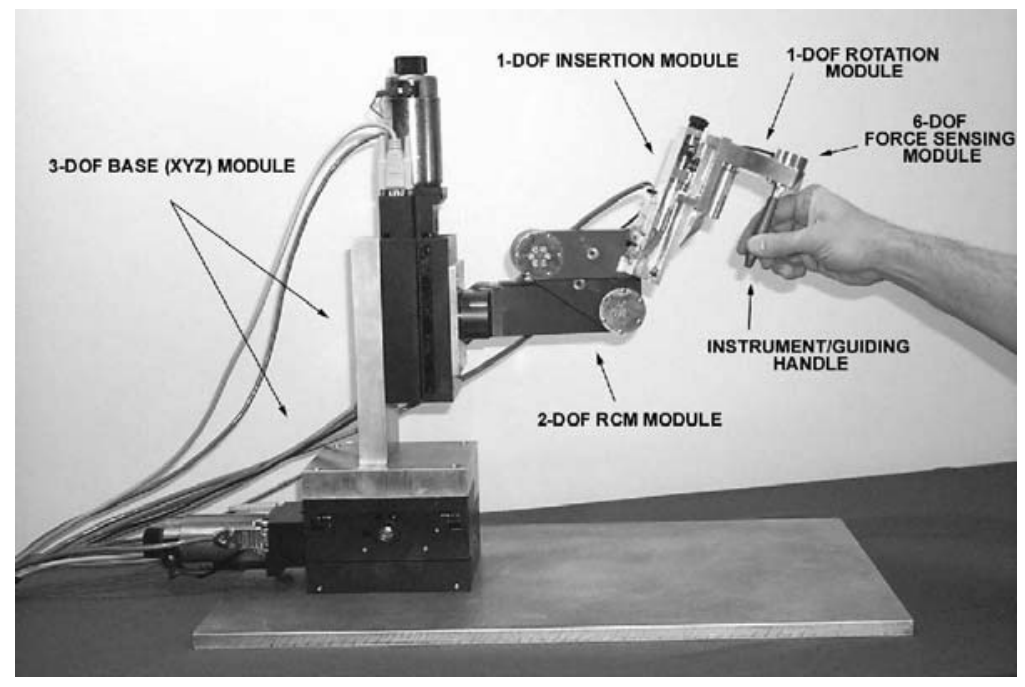

Fig. 1. Steadyhand Surgical Assistant Robot

system can significantly reduce the effects of hand tremor, hold an instrument in a fixed position as a third hand, enforce safety limits on the instrument motion, and provide amplified force reflection between the user manipulation forces and the tool tip contact forces. Our steady-hand surgical assistant robot provides a stable platform for smooth controlled motion of a microsurgical instrument with micrometer level resolution, directly controlled by the operator's manipulation forces and torques on the instrument.

The advantages of cooperative "steady-hand" manipulation over master-slave robot teleoperation systems for microsurgery such as the RAMS system [6] and Intuitive Systems' Da Vinci system [7] are direct manipulation, reduced system cost, and reduced complexity. Since the surgeon holds the actual instruments which operate on the patient to perform procedures using the steady-hand assistant, "steady-hand" assisted surgery is more similar to conventional microsurgery and would require less training by the surgeon to adapt to the system. Steady-hand manipulation requires a single robot system only, rather than the two separate robotic systems required in master-slave teleoperation with force reflection. The advantages of master-slave teleoperation systems over steadyhand manipulation for surgery are that motions can be scaled as well as forces and procedures can be performed remotely.

For precise scaled force reflection, both user manipulation and instrument tip interaction forces must be sensed independently. A commercial six-axis force sensor is used to measure the user's forces and torques on the instrument handle (ATI Industrial Automation Nano-17). At present, however, commercial force sensors are not available with the small size and high resolution necessary to measure microsurgical instrument tool tip forces. To provide the desired enhanced force feedback, we have developed a new sensor to fit inside a microsurgical in- 
strument handle and measure the 3-D force vector at the instrument tip with sub-millinewton precision. This paper reports the design of this force sensor, its testing, and preliminary force scaling control data.

\section{Steady-Hand Surgical Assistant}

The steady-hand surgical assistant robot has a modular design with a 3 DOF linear translation stage module, a 2 DOF remote center of rotation module, and a final tool insertion and rotation stage [8] as shown in Figure 1. The three base stages move the end effector in the Cartesian $x, y$, and $z$ directions, the remote center-of-motion arm provides rotation about $x$ and $y$ axes intersecting at the instrument outside of the body of the robot, and the final stage provides rotation about and translation along the axis of the surgical instrument. Separate force sensors measure user manipulation forces and torques and tool tip forces.

The actuator drives are geared for slow, precise motions with ratios of 50:1 to 200:1 in rotation and $2 \mathrm{~mm} /$ revolution in translation. A microsurgical instrument such as a retinal pic or needle is mounted on the robot as an end effector. The modular construction of the steadyhand surgical assistant robot allows the separate parts of the robot to easily be adapted to various different applications.

The six-axis force sensor is mounted between the robot and the end effector instrument handle to measure the forces and torques exerted by the user during manipulation. The newly developed smaller sensor between the handle and the instrument tip is used to measure the interaction forces between the instrument and its environment, such as delicate bodily tissues in microsurgery. An 8-axis DSP controller card (Motion Engineering Inc. PCX/DSP) installed in a PC provides fast low level closed-loop PID joint position control for the steadyhand robot. The position control bandwidth of the steadyhand robot is $20-25 \mathrm{~Hz}$.

Since typical desired motions are less than $5 \mathrm{~mm} / \mathrm{sec}$, the robot actuators have high transmission ratios, and the environment is highly compliant, the dynamics of the manipulator can be neglected during operation. Force control is implemented as an added layer above the low-level position control by updating the desired velocity in the joint controllers as follows:

$$
\begin{gathered}
f_{\text {des }}=f_{\text {handle }} / C+f_{\text {off } f e t} \\
\dot{x}_{\text {des }}=K\left(f_{\text {des }}-f_{\text {tip }}\right)
\end{gathered}
$$

where $\dot{x}_{d e s}$ is the desired end effector velocity, $K$ is the force-to-velocity control gain, $C$ is the force scaling factor, $f_{t i p}$ is the sensed force at the instrument tip, $f_{\text {handle }}$ is the user manipulation force sensed on the instrument handle, and $f_{\text {offset }}$ is the desired resting tip force, which would be set to zero during typical manipulation tasks. Given the $20 \mathrm{~Hz}$ closed loop joint positioning bandwidth, it is reasonable in this application to neglect servo tracking error errors and assume that $x=x_{\text {des }}$. 


\section{Prior Work}

Microsurgical force measurement experiments were undertaken by Gupta et al [9], showing that typical forces on microsurgical instrument tips during retinal surgery are less than 7.5 millinewton and below the threshold of the operator's tactile sensitivity. Based on these results we conclude that microsurgeons operate using visual feedback without the influence of any tactile sensing feedback through the instruments. Measurement of the hand tremor variation in the position of handheld microsurgical instruments while being held passively and actively has been performed by Riviere et al $[10,11]$. These studies show a limit in human tool positioning accuracy of 20-40 micrometers during microsurgery.

Preliminary results with "steady-hand" interactive force scaling are reported by Kumar et al [12] with motion along a single axis and using a single axis instrument tip force sensor consisting of a single strain gage pair bonded on both sides of the flattened shaft of an instrument tip in a half bridge arrangement. Force scaling experimental results during contacts with stiff wires and porcine eyes were obtained with a tool tip to user handle scaling factor of 25 .

\section{New Instrument Tip Force Sensor}

The new 3-axis sensor measures forces at the instrument tip provided that there are no additional forces or torques exerted on the instrument shaft. The performance requirements for the new instrument tip force sensor are as follows:

$\begin{array}{ll}\text { Force Range: } & \pm 1.0 \mathrm{~N} \\ \text { Force Resolution: } & 0.05 \mathrm{mN} \\ \text { Maximum Force: } & 5.0 \mathrm{~N} \\ \text { Diameter: } & 12.5 \mathrm{~mm} \\ \text { Instrument Tip Length: } & 40 \mathrm{~mm}\end{array}$

Due to the lever arm of the extended instrument tip, a typical four-beam cross force sensor design is much more sensitive to $x$ and $y$ forces at the tip than to $z$ forces in line with the instrument axis. A compact ring-shaped sensor designed by Diddens et al [13] to fit inside a pen and measure writing forces, also has this drawback of anisotropic insensitivity.

To obtain a tool tip sensor with isotropic sensitivity, we have developed a double cross design with two vertically separated flexure beam crosses as suggested in [14]. The relative sensitivity of this sensor configuration in different directions varies with the vertical separation between the crosses. In this design the sensor sensitivity to axial and nonaxial forces was equalized with a $4.0 \mathrm{~mm}$ separation between the beam crosses. The complete force sensor is a cylinder $12.5 \mathrm{~mm}$ in diameter and $12.25 \mathrm{~mm}$ in height.

When the tip force is perpendicular to the instrument shaft, the flexure beams of the sensor are in nearly uniform tension and compression. Forces parallel to the instrument shaft cause bending strains in the beams which are maximized at the ends of each beam, however. The strain gages are bonded at the outer end 


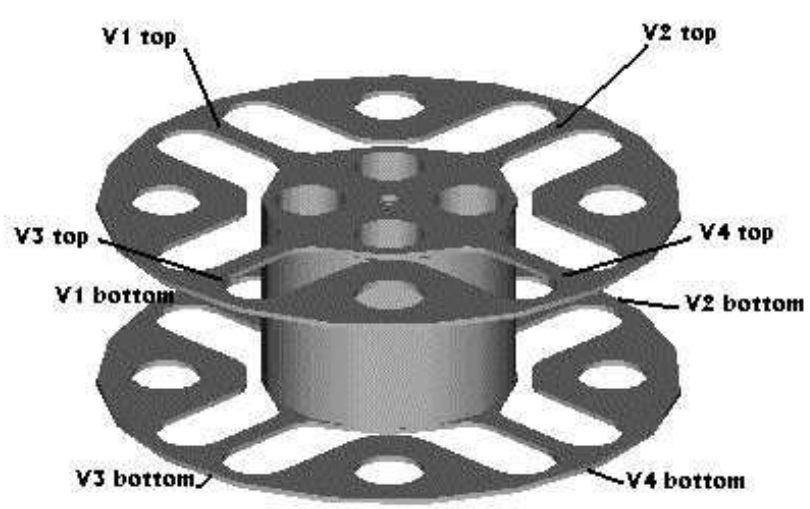

Fig. 2. Strain Gage Configuration

of each flexure beam, above the top set of beams and below the bottom set, as shown in Figure 2. The finite element static strain analysis (FEA) response of the flexure beams of the sensor (I-DEAS Master Series 7, Structural Dynamics Research Corporation) to a $1.0 \mathrm{~N}$ force on the instrument tip in the radial directions is shown in Figure 3, with the strain shown in the bar to the right.

Doped silicon strain gages have a strain gage factor approximately 100 times those of conventional foil gages. Silicon strain gages also have correspondingly greater thermal drift than foil gages, so that we must compensate for thermal effects during operation of the sensor. The parameters of the silicon strain gages used in the force sensor (Micron Instruments, CA) are given below:

$\begin{array}{ll}\text { Size: } & 0.56 \mathrm{~mm} \\ \text { Resistance: } & 500 \Omega \\ \text { Operating Range: } & \pm 2000 \mu \epsilon \\ \text { Maximum Strain: } & \pm 3000 \mu \epsilon \\ \text { Gage Factor: } & 150\end{array}$

The maximum beam strains in the FEA response of Figure 3 are approximately $\pm 500 \mu \epsilon$, well within the operating range of the gages. The safety factors for the strain and shear yield points of the sensor beams are also in the 3-4 range. These safety factors are included to account for residual stresses arising from strain bonding, part fabrication, and sensor assembly.

The first prototype of the new sensor contains eight strain gages in four half bridges. Each gage is paired with its counterpart on the other flexure beam cross. Each pair of strain gages produces a change in voltage at the node between them proportional to the difference in strain. The instrument tip forces are calculated from the strain gage half bridge voltages as follows: 

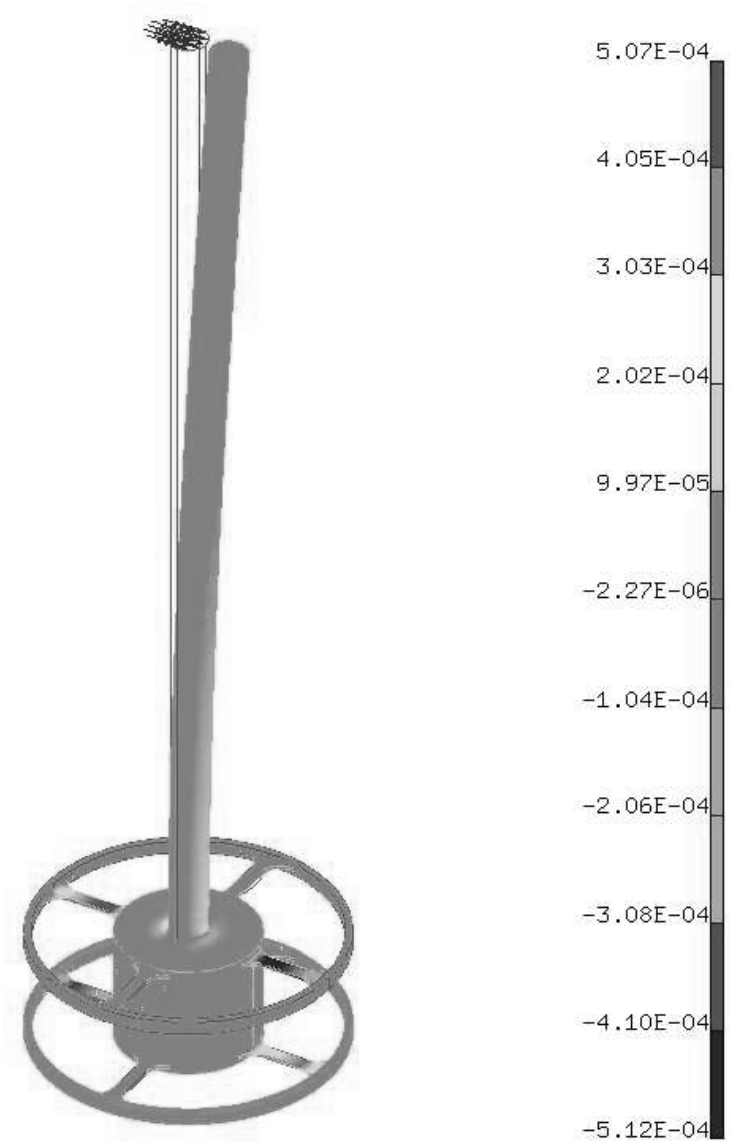

Fig. 3. FEA Force Sensor Strain Response to 1.0 N Radial Force

$$
\begin{gathered}
f_{x} \approx C\left(\Delta V_{1}-\Delta V_{3}\right) / 2 \\
f_{y} \approx C\left(\Delta V_{2}-\Delta V_{4}\right) / 2 \\
f_{z} \approx C\left(\Delta V_{1}+\Delta V_{2}+\Delta V_{3}+\Delta V_{4}\right) / 4
\end{gathered}
$$

where each $\Delta V$ refers to the change in voltage from the unloaded condition for each strain gage pair and $C$ is a scaling factor derived from the gage factor, and sensor beam material and dimensions. To obtain accurate force measurements the sensor must be calibrated to correct for variations in strain gage mounting locations, gage resistances, and beam dimensions.

The voltage signals from the strain gage bridges are amplified by a signal amplifier (Vishay Instruments Division, 2210 A Signal Conditioning Amplifier) with a gain of 100 and digitized by a 16-bit digital to analog converter (ComputerBoards Inc. PCI-DAS1602/16). The maximum resolution of the sensor at 


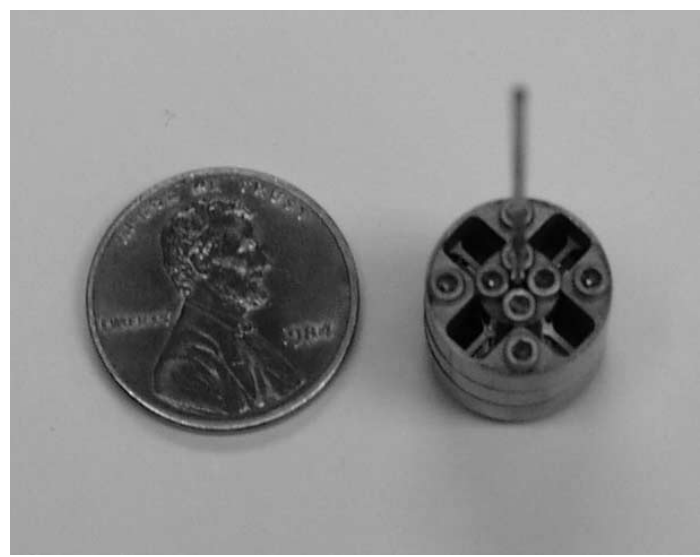

Fig. 4. Fabricated Miniature Force Sensor

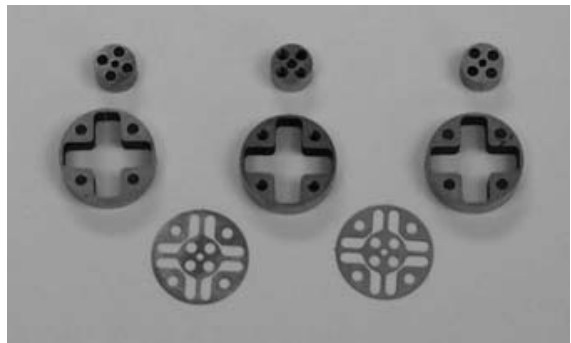

Fig. 5. Force Sensor Components

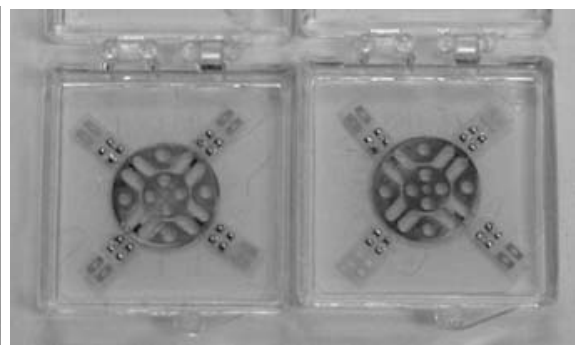

Fig. 6. Flexure Plates with Strain Gages

the $\pm 1.0 \mathrm{~N}$ range due to 16 -bit quantization is 0.015 millinewton in the $x$ and $y$ directions and 0.0075 millinewton in the $z$ direction.

The fabricated force sensor is shown in Figure 4 with a penny for scale. The internal flexure beams are visible. The component parts of the miniature force sensor, shown in Figure 5, were fabricated using wire electrical discharge machining (EDM) to achieve consistent tolerances at submillimeter dimensions and because EDM fabrication does not cause residual stresses, warping, or burrs as may be caused by conventional machining. The flexure plates and other sensor parts are assembled with standard bolts so that the instrument tips and gage plates can be easily replaced in case of damage. The flexure plates of the sensor with bonded strain gages wired to solder pads are shown in Figure 6 .

Since the strain gages and flexure beams in the present sensor may be damaged by tip forces over 5 Newtons, overload limiting will be added to subsequent versions of the tool tip force sensor by placing the sensor inside the instrument handle with a preloaded spring which compresses when applied forces exceed a given threshold, so that the instrument tip is compliant to excessive forces and the sensor will not be damaged. The force sensor beams and wiring will also be enclosed in a small housing to protect against damage from contact with the 


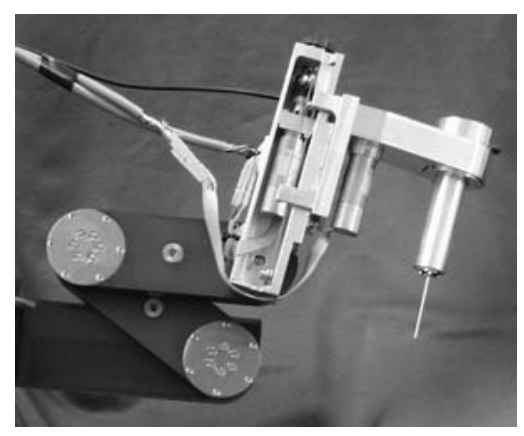

Fig. 7. Steadyhand Force Scaling Setup with New Sensor

environment or operator. Additional strain gages can be bonded to the sensor to form full bridges instead of half bridges, improving sensitivity and greatly reducing thermal drift.

\section{Calibration and Experiments}

The new tip force sensor has been installed at the steadyhand robot end effector with a cylindrical tube as an instrument handle, as shown in Figure 7 . To obtain sensor calibration data, 10, 20, and 50 gram weights were suspended from the tip of the instrument and the rotation stages of the robot were moved so that the sensor was loaded in the $+x,-x,+y,-y$, and $-z$ directions. The amplifiers that were used can internally balance the half bridge arrangements used for the strain gages, so that offsets in the strain gage signals are eliminated. The resulting collection of strain gage bridge signal data can be mapped to the forces by a linear transform which was calculated using a least squares solution:

$$
M=\left[\begin{array}{lll}
0.0360 & 0.6568 & -0.0222 \\
-0.2711 & -0.1086 & -0.0508 \\
0.0236 & -0.2163 & -0.0206 \\
0.1878 & -0.0826 & -0.0536
\end{array}\right]
$$

so that $S M=F$, where $S$ is the vector of sensor gage signal voltages and $F$ is the 3 -D force vector in $\mathrm{N}$. This mapping differs from the expected mapping for the sensor as described in Section 4 due to residual stresses from assembly and material and dimensional variations in the sensor components. These differences can be reduced in subsequent force sensors by assembly under better controlled conditions. The accuracy of the first fabricated sensor is within $\pm 5 \%$ in the tested $\pm 0.5 \mathrm{~N}$ range.

To test steadyhand force scaling control with the new sensor, a latex membrane was suspended horizontally with low tension in the workspace of the robot to simulate bodily tissue. The membrane was palpated with the tip of the force sensor instrument using steadyhand manipulation, with the instrument held by both user and robot. The handle to tip force scaling factor was 30:1 for the tip 


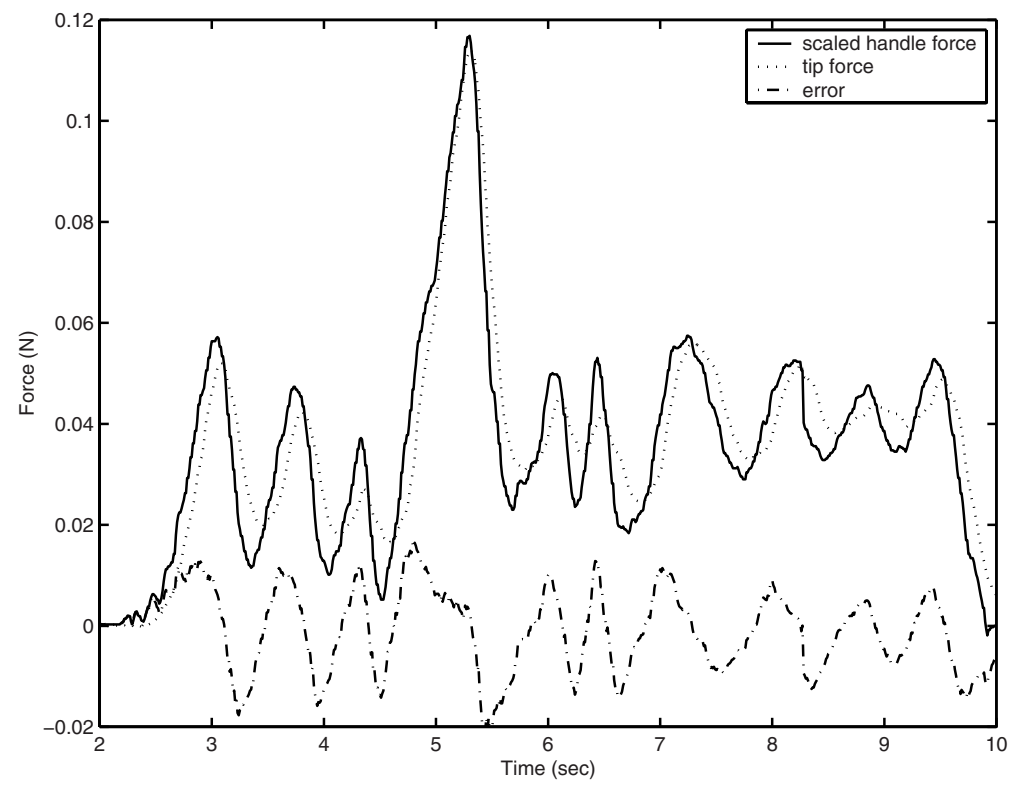

Fig. 8. Surface Contact Force Control Using Miniature Force Sensor

force trajectory shown in Figure 8. The forces shown in Figure 8 are the vertical components of the forces measured by the handle and instrument tip force sensors, with all gages active in the instrument tip sensor. The tip force is a smoother filtered response to the scaled handle force trajectory.

\section{Conclusion}

The new force sensor described will enable steadyhand enhanced feedback force scaling in all directions, not just a single axis as demonstrated in the preliminary experiment. The fidelity of millinewton force control in microsurgery can be greatly improved with the new sensor due to its high resolution. Although the sensor was designed specifically for enhanced feedback during steadyhand cooperative manipulation, use of this miniature precision force sensor will allow instrument tip forces to be measured in other microsurgical procedures.

Preliminary testing of the steadyhand surgical assistant using the tool tip force sensor is planned for application to various eye and ear microsurgical procedures, leading to clinical testing. Other application areas envisioned for the steadyhand surgical assistant system include neurosurgery and microvascular surgery. The system may also be used for applications such as MEMS assembly.

The complete system combines the accuracy of the robot with the sensitivity of the described instrument tip force sensor and the dexterity of the human for better performance of microsurgical tasks. 


\section{References}

[1] R. H. Taylor, J. Funda, B. Eldridge, K. Gruben, D. LaRose, S. Gomory, and M. Talamini, A Telerobotic Assistant for Laparoscopic Surgery, ch. 45, pp. 581592. MIT Press, 1996.

[2] R. H. Taylor, H. A. Paul, P. Kazandzides, B. D. Mittelstadt, W. Hanson, J. F. Zuhars, B. Williamson, B. L. Musits, E. Glassman, and W. L. Bargar, "An imagedirected robotics system for precise orthopedic surgery," IEEE Transactions on Robotics and Automation, vol. 10, no. 3, pp. 261-275, 1994.

[3] J. Troccaz, M. Peshkin, and B. L. Davies, "The use of localizers, robots and synergistic devices in cas," in First Joint Conference of CVRMed and MRCAS, (Grenoble, France), pp. 727-729, 1997.

[4] S. J. Harris, W. J. Lin, K. L. Fan, R. D. Hibberd, J. Cobb, R. Middleton, and B. L. Davies, "Experiences with robotic systems for knee surgery," in First Joint Conference of CVRMed and MRCAS, (Grenoble, France), pp. 727-729, 1997.

[5] S. C. Ho, R. D. Hibberd, and B. L. Davies, "Robot assisted knee surgery," IEEE EMBS Special Issue on Robotics in Surgery, pp. 292-300, April 1995.

[6] S. Charles, "Dexterity enhancement for surgery," in First International Symposium on Medical Robotics and Computer Assisted Surgery, vol. 2, pp. 145-160, 1994.

[7] G. S. Guthart and J. K. Salisbury, "The intuitive(tm) telesurgery system: Overview and application," in IEEE International Conference on Robotics and Automation, (San Francisco), pp. 618-621, April 2000.

[8] R. H. Taylor, P. Jensen, L. Whitcomb, A. Barnes, R. Kumar, D. Stoianovici, P. Gupta, Z. Wang, E. deJuan, and L. Kavoussi, "A steady-hand robotic system for microsurgical augmentation," in Medical Image Computing and ComputerAssisted Interventions (MICCAI), (Cambridge, England), pp. 1031-1041, September 1999.

[9] P. K. Gupta, P. S. Jensen, and E. deJuan Jr., "Surgical forces and tactile perception during retinal microsurgery," in Medical Image Computing and ComputerAssisted Interventions (MICCAI), (Cambridge, England), pp. 1218-1225, September 1999 .

[10] M. Gomez-Blanco, C. Riviere, and P. Khosla, "Intraoperative instrument motion sensing for microsurgery," Proc. of the 21st Conf. of the IEEE Engineering in Medicine and Biology Society, p. 864, October 1999.

[11] C. N. Riviere, P. S. Rader, and P. K. Khosla, "Characteristics of hand motion of eye surgeons," in Proc. of the 19th Conf. of the IEEE Engineering in Medicine and Biology Society, (Chicago), pp. 1690-1693, October 1997.

[12] R. Kumar, P. Berkelman, P. Gupta, A. Barnes, P. Jensen, L. Whitcomb, and R. Taylor, "Preliminary experiments in cooperative human/robot force control for robot assisted microsurgical manipulation," in IEEE International Conference on Robotics and Automation, (San Francisco), pp. 610-617, April 2000.

[13] D. Diddens, D. Reynaerts, and H. van Brussel, "Design of a ring-shaped threeaxis micro force/torque sensor," Sensors and Actuators, vol. A46, pp. 225-232, January 1995.

[14] D. Reynaerts, Piers, and H. van Brussel, "A mechatronic approach to microsystem design," IEEE/ASME Transactions on Mechatronics, vol. 3, pp. 24-33, 1998. 